\title{
IDENTIFICATION OF DNA OF PATHOGENS FROM Fusarium GENUS IN MAIZE PLANTS (Zea mays L.)
}

\author{
Tumanova Lidia, Grajdieru Cristina, Mitin V. \\ Institute de Genetics, Physiology and Plant Protection, Chisinau, Republic of Moldova \\ e-mail: lidia tumanova@yahoo.com
}

Fungal infestation of maize fields with Fusarium species leads to yield loss and affects grain quality. Main factors that define grain infection rate are species composition of Fusarium microflora, host-plant genotype and grade of it's resistance. Main interest present Fusarium species that produce mycotoxins, which can be present in unsafe background concentrations in symptomless of affected grain. That is why plant state monitoring and early diagnostic have an important significance for preventing pathogen dissemination. PCR-based assays of molecular diagnostic allow fast and precise phytopathogens identification, including cases of mixed contamination, differentiate species that provoke similar symptoms, minimize the risks of pathogen dissemination during work process. Current paper outlines the results of Fusarium spp. DNA identification on several maize genotypes.

Nested-PCR for Fusarium fungi identification was performed using primers designed for amplification of TEF1 gene partial sequence common for species of Fusarium genus and TEF1 gene partial sequence specific for $F$. oxysporum, $F$. avenaceum, $F$. verticillioides. Maize plants of 3 genotypes were analyzed for mixed contamination with Fusarium fungi. Samples of bottom part of the cobs at the silking stage, kernels at the dough stage and stage of physiological maturity were analyzed. For enhancing the efficiency of simultaneous phytopathogen detection parameters of multiplex-PCR were optimized (temperature of alignment, primer mix, number of cycles in the first and second round). Primarily, DNA samples were analyzed for presence of Fusarium spp. In case of positive signal (apparition of specific amplicons corresponding to $F$. spp.) samples were further analyzed for differentiation of Fusarium species. In the same way simultaneous detection of $F$. avenaceum, $F$. oxysporum, $F$. verticillioides was performed. Molecular analysis demonstrates that propagation of fungi depends on plant genotype and phase of development. Fusarium species were not identified in cobs prior to silk emerging. Mass infestation of plants was ascertained at the dough stage. Analyzed genotypes showed a different grade of infection for certain Fusarium species at the dough stage. Mixed infections of $F$. oxysporum, $F$. avenaceum, $F$. verticillioides were detected in all 'KY123' samples and in several samples of 'RF7' genotype. Molecular analysis as well showed that 'MK01' genotype was infected only with $F$. oxysporum, but infection rate constituted $30 \%$ of 'KY123' and 'RF7' infection rate (100\%). Mixed infection of $F$. oxysporum, F. avenaceum, $F$. verticillioides was identified in about all samples of mature kernels of the studied genotypes. Obtained data allows to presume that 'MK01' is the most resistant to Fusarium spp. among the three genotypes. Analyzing of cob bottom part at the dough phase can be proposed as reliable method for corn genotype resistance estimation to Fusarium species. Outlined approach can be used for corn varieties and commercial hybrids' screening based on their susceptibility to Fusarium fungi. 